\title{
DMSO exhibits similar cytotoxicity effects to thalidomide in mouse breast cancer cells
}

\author{
ECE SIMSEK ÖZ $^{1,2}$, ESRA AYDEMIR $^{1,2}$ and KAYAHAN FIŞKIN ${ }^{1}$ \\ ${ }^{1}$ Department of Biology, Art and Science Faculty, Akdeniz University, Antalya 07058; \\ ${ }^{2}$ Central Research Laboratories and Immunology, School of Medicine, Akdeniz University, Antalya 07070, Turkey
}

Received August 1, 2011; Accepted November 3, 2011

DOI: $10.3892 / 01.2012 .559$

\begin{abstract}
The purpose of this study was to evaluate the cytotoxic effect of thalidomide on 4T1 and 4THMpc mouse breast cancer cell lines. Mouse breast cancer cells (4T1) and cells derived from metastatic lesions (4THMpc) were treated with various doses of thalidomide $\left[10^{-2}-100 \mu \mathrm{M}\right.$ dissolved in dimethyl sulfoxide (DMSO) as recommended] and $1.4 \mu \mathrm{M}$ DMSO (maximum DMSO concentration in the highest thalidomide dose) as a DMSO control against the untreated control groups. MTT was used to evaluate the cytotoxic effects of the treatments. Therefore, we investigated the role of thalidomide on apoptosis. A fluorometric EnzChek caspase-3 enzyme activity assay kit was used to evaluate the apoptotic effects of thalidomide. Thalidomide dissolved in DMSO exhibited cytotoxic effects on 4T1 and 4THMpc cells compared to the control groups incubated without any supplement. Treatment with thalidomide resulted in apoptosis of mouse breast cancer cells in a time- and dose-dependent manner as demonstrated by caspase-3 enzyme activity. However, DMSO alone suppressed cell proliferation more effectively than thalidomide. In cultured mouse breast cancer cells the inhibitory effect of thalidomide may be partially attributed to the solvent DMSO alone.
\end{abstract}

\section{Introduction}

Breast cancer, which is the second leading cause of cancer mortality in women, occurs at a high frequency (1). Although there have been significant advances in diagnosing and treating breast cancer, a number of major unresolved clinical and scientific problems remain and investigations are ongoing (2).

Thalidomide $[\mathrm{a}(\mathrm{N}$-phthalimido)-glutarimide, a derivative of glutamic acid with two rings and two optically active forms] is an odorless, white crystalline compound with low solubility in water and has a cytotoxic effect on a number of cancer cell lines, including breast cancer $(3,4)$.

Correspondence to: Dr Ece Simsek Öz, Department of Biology, Art and Science Faculty, Akdeniz University, Antalya 07058, Turkey E-mail: ece0708@hotmail.com

Key words: DMSO, thalidomide, 4T1, 4THMpc, apoptosis
Thalidomide has been shown to be clinically useful in a number of situations due to its ability to inhibit TNF- $\alpha$ synthesis. However, its use is restricted by potentially serious side effects, including teratogenicity (5). Furthermore, insolubility may cause serious problems in terms of systemic bioavailability. The major solvent of thalidomide is dimethyl sulfoxide (DMSO) and it has been shown to mimic the cytotoxic effects of thalidomide (6).

The aim of this study was to evaluate the effect of thalidomide and DMSO on the viability of cultured 4T1 and 4THMpc mouse breast cancer cell lines.

\section{Materials and methods}

Thalidomide. Thalidomide was purchased from SigmaAldrich (Cat. No. T 144; St. Louis, MO, USA). A $100 \mu \mathrm{M}$ stock solution dissolved in DMSO $(14.08 \mathrm{mM})$ was prepared and then the solution was aliquoted into standard Eppendorf tubes in quantities of $500 \mu \mathrm{l}$ for daily assays. These aliquots were stored at $-70^{\circ} \mathrm{C}$ until required.

Cell lines and in vitro cell culture conditions. 4T1 breast cancer cells and 4T1 heart metastases post capsaicin (4THM), a cell line obtained from orthotopically transplanted 4T1 breast cancer cells, were used in this study. The cell lines were a kind gift from Dr Nuray Erin (Akdeniz University, Medicine Faculty, Antalya, Turkey). Cells were grown as monolayer adherent cultures in plastic cell culture petri dishes (BD, Bedford, MA, USA) in DMEM-F12 (Biochrom, Germany) supplemented with $5 \%$ fetal bovine serum (FBS), $2 \mathrm{mM}$ L-glutamine, $1 \mathrm{mM}$ sodium pyruvate and $0.02 \mathrm{mM}$ non-essential amino acids. The cell lines were maintained at $37^{\circ} \mathrm{C}$ in a humidified atmosphere of $5 \% \mathrm{CO}_{2}$. The cell lines used in this study were tested and shown to be free of mycoplasma contamination.

Thalidomide and DMSO treatments. To determine the effects of the cell number on the cytotoxic effects of thalidomide and DMSO, initial experiments were performed with three different cell numbers (1,000, 3,000 and 5,000 cells/well) and 5,000 cells/well was selected for all experiments.

Cells were seeded and $24 \mathrm{~h}$ later treated with thalidomide $\left(10^{-2}-100 \mu \mathrm{M}\right.$ dissolved in DMSO) and 1.4 $\mu \mathrm{M}$ DMSO alone. The fresh medium was added to the control groups. Following the treatments, the cells were incubated for three different 
incubation periods (24, 48 and $72 \mathrm{~h})$ and then the cell growth was determined.

Determining cell viability. Cell proliferation was determined using thetetrazoliumcompound3-(4,5-dimethylthiazol-2-yl)-5(3-carboxymethoxyphenyl)-2-(4-sulphophenyl)-2H-tetrazolium, inner salt (MTS) according to the manufacturer's instructions (Cell Titer 96 Aqueous One Solution Cell Proliferation Assay; Promega Corp., Madison, WI, USA). Formation of formazan determined at OD 490 was compared between the groups. In this study, one group was treated with thalidomide $(100 \mu \mathrm{M})$, and the other group was treated with DMSO. The control group received refreshed medium containing $1 \%$ serum.

Fluorometric caspase-3 enzyme activity assay. Caspase-3 activity in total cell lysates was determined by a fluorometric EnzChek caspase-3 assay kit (Molecular Probes, Eugene, OR, USA) using 7-amino-4-methylcoumarin-derived substrate Z-DEVD-AMC, according to the manufacturer's instructions. Briefly, thalidomide and DMSO were administered to the wells ( 200,000 cells/well in a 6-well plate). After $48 \mathrm{~h}$, the cells were harvested using trypsinization and cell lysates were prepared. The protein concentration of the samples was determined using a Bio-Rad protein assay kit (Bio-Rad Laboratories, Hercules, CA, USA) and measured against bovine serum albumin standards. Samples of the cell lysates were seeded into standard black 96-well plates as each sample contained $10 \mu \mathrm{g}$ protein per well. Samples were mixed with reaction buffer and the plate was incubated for $2 \mathrm{~h}$ at room temperature avoiding direct light. Then, $200 \mu \mathrm{mol} / \mathrm{l}$ substrate of caspase-3, Z-DEVD-AMC, was added to each well and incubated for $1 \mathrm{~h}$ at $37^{\circ} \mathrm{C}$ in the dark. The assay conditions were standardized in order that the products of the reaction remained in the linear range of detection. The plate was detected by a fluorescence spectrophotometer using 496/520 $\mathrm{nm}$ excitation/emission wavelengths and the sample readings were calculated by subtracting the absorbance of blank samples.

Statistical analysis. Data were expressed as the means \pm SEM and analyzed statistically using one-way ANOVA followed by Dunnett's multiple comparisons test for comparison of the group means to controls (Graph Pad InStat., USA). P $<0.05$ was considered statistically significant.

\section{Results}

In vitro cytotoxic effects of thalidomide dissolved in DMSO and DMSO alone on $4 T 1$ and $4 T H M p c$ cell lines. Results obtained from the experiments were statistically analyzed and are shown in Figs. 1-3. As shown in Fig. 1, DMSO was more cytotoxic than thalidomide on 4T1 cells. This cytotoxic effect was time-dependent. Time 0 indicates the cell number at the beginning of the experiment. The same results obtained from the experiments carried out on 4THMpc cells are shown in Fig. 2. The cytotoxic effect of DMSO on 4THMpc cells was also time-dependent.

Using a fluorometric assay, we determined whether incubation of cells with thalidomide and DMSO for $48 \mathrm{~h}$ was capable of inducing caspase-3 activity. The results are shown

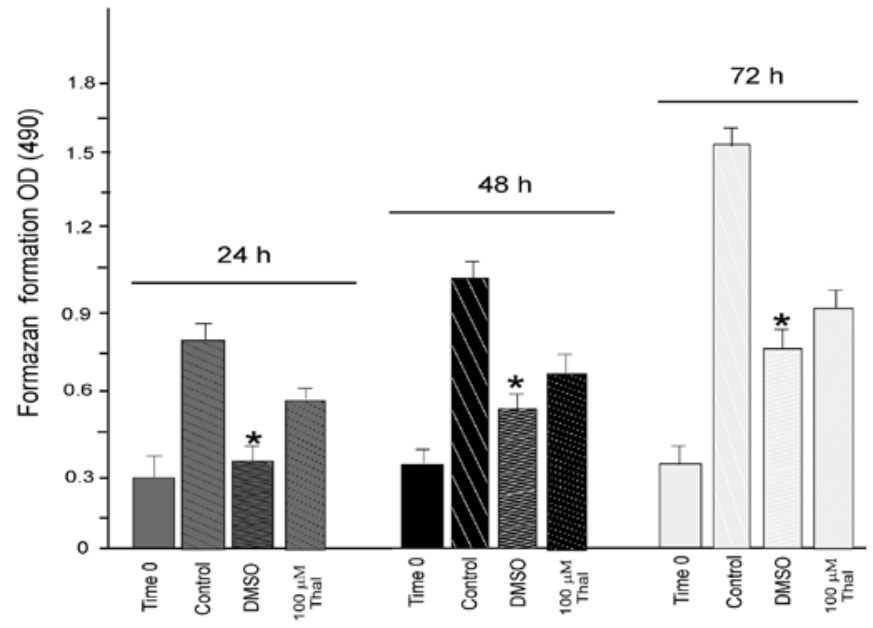

Figure 1. In vitro cytotoxic effects of DMSO and thalidomide on 4T1 mouse breast cancer cells. DMSO $(1.4 \mu \mathrm{M})$ and thalidomide $(100 \mu \mathrm{M})$ exhibit cytotoxic effects on the $4 \mathrm{~T} 1$ mouse breast cancer cell line. Time 0 indicates the cell number at the beginning of the experiment. Cells treated with any supplement were evaluated as the control group.

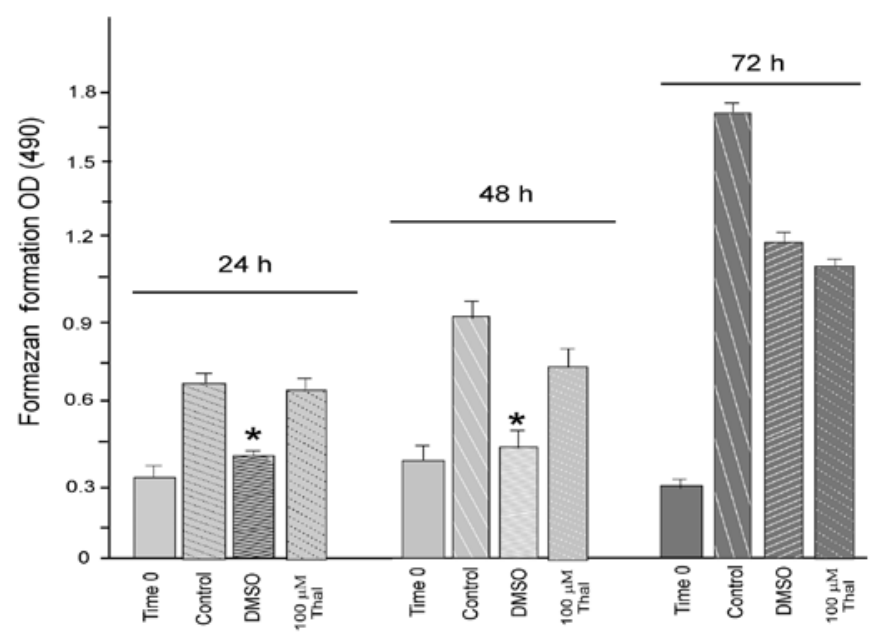

Figure 2. In vitro cytotoxic effects of DMSO and thalidomide on 4THMpc mouse breast cancer cells. DMSO $(1.4 \mu \mathrm{M})$ and thalidomide $(100 \mu \mathrm{M})$ exhibit cytotoxic effects on the 4THMpc mouse breast cancer cell line. Time 0 indicates the cell number at the beginning of the experiment. Cells treated with any supplement were evaluated as the control group.

in Fig. 3. In the two cell lines, both DMSO and thalidomide triggered caspase- 3 activity compared with the control groups. The increase in caspase- 3 activity was markedly prevented following combined treatment by thalidomide and DMSO together with the broad caspase inhibitor zVAD-fmk.

\section{Discussion}

The first aim of this study was to test the cytotoxic effects of thalidomide on mouse breast cancer cells 4T1 and 4THMpc. Breast cancer is a life-threatening disease that may be cured by pharmacological therapies.

Thalidomide widely used in the treatment of cancer in its present form is not soluble in water, and for in vitro experiments DMSO is commonly used as a solvent (6). Thalidomide 


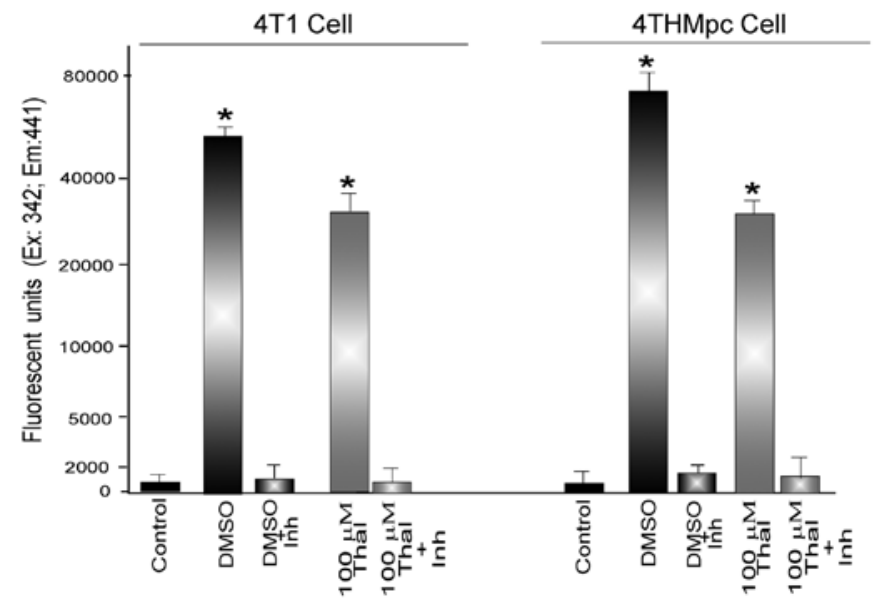

Figure 3. Changes in caspase-3 enzyme activity. DMSO $(1.4 \mu \mathrm{M})$ and thalidomide $(100 \mu \mathrm{M})$ caused a significant increase in caspase-3 enzyme activity. This effect was blocked by adding caspase-3 inhibitor.

(1 mM) dissolved in $14.08 \mu \mathrm{M}$ DMSO was used as a stock solution. DMSO $(1.4 \mu \mathrm{M})$ alone was used as a control.

Our results showed a cytotoxic effect of $100 \mu \mathrm{M}$ thalidomide dissolved in DMSO on mouse breast cancer cells compared to the control cells without any supplement. Therefore, we showed that DMSO alone in a concentration equivalent to dissolved thalidomide was capable of exhibiting cytotoxic effects as well.

Only a few studies have used thalidomide in in vitro experiments. In two studies, Kaven et al and Spraul et al indicated that thalidomide dissolved in DMSO showed an inhibitory effect on the migration and proliferation of rabbit pigment epithelium (RPE) cells compared to controls without any supplement $(7,8)$. However, these authors did not use DMSO alone as a control.

By contrast, Moreira et al demonstrated a dose-dependent inhibition of human umbilical vein endothelial cell proliferation by thalidomide solved in DMSO. These authors used DMSO alone and obtained no significant results on inhibition (9). In their study, Bauer et al used DMSO alone as a control and DMSO did not exhibit an inhibitory effect (10).

At present, thalidomide is used for its anti-angiogenic effect, but the exact mechanism of this property remains to be elucidated. The possible cause may be the action of one of its metabolites. The same may be true for DMSO. Of note, water-soluble thalidomide has recently been produced.

However, further the cell culture experiments with thalidomide are required to confirm these results.

In conclusion, we showed a cytotoxic effect of thalidomide dissolved in DMSO on 4T1 and 4THMpc mouse breast cancer cell lines. Therefore, this effect may partially be attributed to the solvent DMSO alone.

\section{Acknowledgements}

This sutdy was supported by the Scientific and Technological Research Council of Turkey (TÜBİTAK project no: 104 T 204). We would like to thank all the employees of the Akdeniz University Scientific Research Project Unit.

\section{References}

1. Deng CX: Survey and summary BRCA1: cell cycle checkpoint, genetic instability, DNA damage response and cancer evolution. Nucleic Acids Res 34: 1416-1426, 2006.

2. Polyak K: Breast cancer: origins and evolution. J Clin Invest 117: 3155-3163, 2007.

3. Singhal S and Mehta J: Thalidomide in cancer. Biomed Pharmacother 56: 4-12, 2002.

4. Stebbing J, Benson C, Eisen T, et al: The treatment of advanced renal cell cancer with high-dose oral thalidomide. $\mathrm{Br} \mathrm{J}$ Cancer 85: 953-958, 2001.

5. Marriott JB, Westby M, Cookson S, et al: CC-3052: A water-soluble analog of thalidomide and potent inhibitor of activation-induced TNF-\{alpha\} production. J Immunol 161: 4236-4243, 1998.

6. Eter $\mathrm{N}$ and Spitznas M: DMSO mimics inhibitory effect of thalidomide on choriocapillary endothelial cell proliferation in culture. Br J Ophthalmol 86: 1303-1305, 2002.

7. Kaven C, Spraul CW, Zavazava N, et al: Thalidomide and prednisoslone inhibit growth factor-induced human retinal pigment epithelium cell proliferation in vitro. Ophthalmologica 215: 284-289, 2001.

8. Spraul CW, Kaven CK, Kampmeier JK, et al: Effect of thalidomide, octreotide, and prednisolone on the migration and proliferation of RPE cells in vitro. Curr Eye Res 19: 483-490, 1999.

9. Moreira AL, Friedlander DR, Shif B, et al: Thalidomide and a thalidomide analogue inhibit endothelial cell proliferation in vitro. J Neurooncol 43: 109-114, 1999.

10. Bauer KS, Dixon SC and Figg WD: Inhibition of angiogenesis by thalidomide requires metabolic activation, which is species-dependent. Biochem Pharmacol 55: 1827-1834, 1998. 